\title{
The real cost of care: focus on value for money, rather than price-tags
}

\author{
Janet Martin, PharmD • Davy Cheng, MD
}

Received: 6 July 2015/Revised: 9 July 2015 / Accepted: 16 July 2015/Published online: 15 August 2015

(c) The Author(s) 2015. This article is published with open access at Springerlink.com

The likely basis of our natural aversion to cost-saving initiatives stems from fears that costs will override considerations for effectiveness and that costs are an unfair, biased, and distasteful frame of reference for guiding clinical decisions where life and limb are at stake. Yet, blanket prohibition of cost considerations in clinical decision-making is a ticket to disaster and is based on naïve assumptions that healthcare resources are unlimited. In fact, ignoring costs will result in more loss of life and limb than a reasoned approach to considering value for dollar, since a "free-for-all" approach to decision-making results in less value extracted from our given resources.

Resources in healthcare are limited, and as a result, choices must be made about what to use and what to forgo in providing medical services. Even if we could eliminate all wasteful practices and limit ourselves only to the interventions in healthcare that "work", we would still face the quandary that resources are insufficient to meet all needs. Again, this requires making choices about what to include and what to leave out. Ideally, these decisions will be made based on the best available scientific data about what "works" (i.e., evidence-based medicine) and what

J. Martin, PharmD - D. Cheng, MD

Centre for Medical Evidence, Decision Integrity \& Clinical

Impact (MEDICI), Western University, London, ON, Canada

J. Martin, PharmD · D. Cheng, MD ( $\varangle)$

Department of Anesthesia \& Perioperative Medicine, Western

University, London, ON, Canada

e-mail: davy.cheng@1hsc.on.ca

J. Martin, PharmD

Department of Epidemiology \& Biostatistics, Western

University, London, ON, Canada range of options will offer the most value for patients from within our set of available resources.

\section{Role of health economic analysis}

To appreciate cost containment initiatives, we need to understand the foundational metrics of health economics: cost-minimization, cost-effectiveness, cost-utility, and cost-benefit analysis. ${ }^{1}$ In particular, we need to understand that costs and cost analyses are not bona fide health economic analyses since they provide just one side of the equation, i.e., costs only. In healthcare, costs alone are not enough; we are often willing to pay increasingly more for increasingly better outcomes. Hence, we are concerned with costs (C) and effects (E) and, more to the point, whether the increased costs are worthy of the added benefits. Health economic analysis involves calculating a value equation to estimate the cost-effectiveness of alternative options, either through cost-effectiveness analysis (i.e., the incremental cost required to achieve an incremental benefit for drug $\mathrm{A} v s$ drug $\mathrm{B}$, expressed as $\Delta \mathrm{C} /$ $\Delta \mathrm{E})$ or through cost-utility analysis (i.e., the incremental cost required to achieve an incremental quality-adjusted life year for drug A $v s$ drug B expressed as $\Delta \mathrm{C} / \Delta \mathrm{QALY}){ }^{1}$ Cost-minimization analysis, on the other hand, is a special type of cost-effectiveness analysis where the alternatives are considered equivalent (and hence $\Delta \mathrm{E}$ cancels out to zero), and only the difference in costs remains relevant $(\Delta \mathrm{C})$. In this special case of equivalence, costs alone suffice for decisions about cost-effectiveness. For cost minimization analysis, the difficulty arises in proving equivalence or at least proving that there are no differences between the drugs that are big enough to be clinically relevant. 
With these principles in mind, we reflect on the study by Tabing et $a l^{2}$ in this issue of the Journal. Their cohort study describes the effect of limited access to three relatively higher cost intraoperative drugs (remifentanil, dexmedetomidine, and desflurane) on anesthesia drug costs and on selected perioperative outcomes for all surgical procedures with available data. Data were compared retrospectively across two cohorts: one prior to limited access to desflurane (through a convenience cohort defined by unobstructed availability within the OR setting prior to a temporary manufacturer recall of desflurane vaporizers) and one following the study intervention to reduce access to costly anesthetic drugs (by inconvenience, requiring the pharmacy to take the additional step of noting the attending anesthesiologist for the case prior to release of remifentanil, dexmedetomidine, or desflurane). The manufacturer's withdrawal of desflurane was used as a trigger for an intervention to reduce access to this more costly inhalational anesthetic as well as two other costly intravenous agents (remifentanil and dexmedetomidine) for which less costly alternatives arguably exist. As expected, reducing ease of access for these more costly agents resulted in decreased utilization and anesthetic drug cost savings with no measurable effect on clinical outcomes or hospital length of stay.

No doubt, imposing barriers to access to cost-prohibitive drugs will generally be expected to decrease utilization and drug costs. Nevertheless, before wholesale acceptance of applying this intervention in other settings, a number of potential reservations should be addressed: Is restriction appropriate and ethical? Is it worth it? How can we proactively turn our attention to "value" rather than to cost alone?

\section{Is it appropriate to restrict costly drugs?}

Restricting access evokes a natural aversion in most clinicians-after all, how can such "administrative measures" ever predict all patient situations that we as clinicians must navigate? Surely, some patients who would benefit from the restricted drug will now be denied. Yet pharmacy formularies routinely use this approach (with proven effects on drug costs for hospitals and governmentfunded drug programs). ${ }^{3,4}$ Furthermore, most practitioners are not inherently familiar with costs, let alone the costeffectiveness of the range of drugs from which they choose. So, while we may naturally advocate for free and unrestricted access to all drugs to allow unrestricted choice for our patients, such unrestricted access presumes that we have the ability to choose wisely amongst the alternatives provided. In reality, however, we choose without the necessary information to do so, i.e., without knowledge of the proven added benefit and incremental cost of successively more costly agents over available alternatives.

The availability of a product on the market neither infers that it automatically represents an advance above available alternatives nor suggests that it is good value for money and hence should be automatically available for use in the hospital setting. Existence of a drug on the market means only that it has been deemed suitable for the market and not that it is necessarily better or "worth it" compared with alternatives. Restriction of higher cost drugs may be reasonable when (1) the added benefit of the costlier drug has not been proven; (2) a margin of benefit has been proven but is too small to warrant the additional cost; and (3) restriction can be overcome when warranted (such as during drug shortages or when a special clinical situation arises that is truly justified). The first condition is easy to understand when taken only at face value, but it becomes a challenge when we consider that clinical trials are often underpowered to prove that "no difference" exists, and can state only that "no difference was found". In this case, "absence of evidence is not evidence of absence". 5 In other words, a lack of studies with adequate power and study design to rule out clinically relevant differences between drugs does not automatically mean that the drugs are equivalent. It means only that they have not been proven to be different. Whether or not they provide different outcomes cannot be known until a sufficient number of studies have been performed to provide narrow enough confidence intervals to rule out differences that are big enough to be worthy of consideration.

In the study by Tabing et al., ${ }^{2}$ the decision to restrict access to the three drugs was presumably based on a combined consideration of cost plus no proven benefit over less costly available alternatives (or insufficient proof of benefits large enough to warrant the significantly greater cost). Randomized trials and meta-analyses of remifentanil, dexmedetomidine, and desflurane in the operating room (OR) setting have generally failed to prove their superiority over cheaper alternatives. ${ }^{6-14}$ In addition, the authors instituted a minimal barrier to prevent access to the three drugs (i.e., by keeping the drugs out of easy reach and making note of the attending anesthesiologist before releasing the drug from the OR pharmacy). It remains uncertain whether this modest barrier would be sufficient to sustain reductions in inappropriate utilization over time.

\section{Is it ethical to restrict costly drugs?}

Ethical objections to restricting access to drugs are difficult to argue when there is a lack of definitive proof that the more expensive drug delivers added benefit. Arguments 
might still be made for one-off "exceptional" cases where evidence is lacking due to rarity and where a single patientspecific use of the drug might be warranted when reasonable alternatives fail or are contraindicated.

Ethical objections may also arise from the perspective of the teaching institution. That is, can we justify limiting access to newer drugs if teaching is our mandate? In a teaching centre, there is some value in maintaining access to a variety of anesthetic drugs for education and research purposes. Since these aspects are not usually considered in cost-effectiveness analyses, we should find ways to incorporate these "intangibles" more explicitly into our assessment of restriction-related impacts. When pondering these aspects, consideration should also be given to the counterfactual issues of using the opportunity to teach trainees to participate in ensuring good stewardship of resources and to navigate a system where only selected drugs are available. In addition, the constantly expanding base of drugs, devices, and technology has created a situation where we cannot expect continuous exposure (both now nor in the future) to all such relevant innovations. Rather than try to maintain exposure to costineffective agents on behalf of our trainees, perhaps we should consider a new paradigm. Trainees and clinicians can learn to keep pace with a rapidly changing environment as well as gain skills and knowledge about inaccessible agents through the use of proactive or on-demand video training and innovative simulation training. Since new interventions are constantly entering the market, this "problem" of lack of exposure will only worsen with time.

Driving the cost of anesthesia drugs to their lowest overall denominator in perioperative surgical care may have had unintended consequences, such as contributing to the drug shortages over the past decade. ${ }^{15}$ For example, older drugs are often most cost effective from the hospital perspective; however, from the manufacturer's perspective, these older drugs are the least cost effective in a for-profit business. Furthermore, in a free enterprise society, governments cannot enact laws that force companies to manufacture products. As a result, generic injectable medications and anesthesia drugs are vulnerable to supply disruption and drug shortage due to market concentration. Notable examples are the recent cases of Sandoz and Hospira, suppliers of $80 \%$ of the generic injectable medications used in the hospital setting. ${ }^{16}$ For industry, a new drug can take ten to 15 years to develop, with an average cost (including failures) of over \$2.6 billion. ${ }^{17}$ Development of anesthesia drugs is often considered low priority by industry given more lucrative return-on-investment from non-anesthetic medications for certain diseases. On balance, rather than drive prices to near zero, we need to consider that a key element to spur and sustain innovation is to pay what the drug is worth.

\section{Is it "worth it" to restrict?}

In order to be considered worthy, the cost savings achieved must be worth more than whatever is forgone, including the reduced freedom of choice and any extra costs and efforts required to implement and maintain the cost-saving intervention. While it is common knowledge that surgery is a costly endeavour when direct costs are considered as a proportion of total hospital expenditure, few formal analyses of operating room costs have been published. In a study of surgical-related costs performed in the United States private healthcare setting, operating rooms accounted for $40 \%$ of total hospital expenses while generating $70 \%$ of the revenue. ${ }^{18}$ Drug costs (anesthetic and non-anesthetic) accounted for $6 \%$ of total hospital costs, $22 \%$ of which accounted for anesthetic drugs. ${ }^{18}$ Nevertheless, when total OR costs were considered, intraoperative anesthesia drug costs accounted for only $5.6 \%$ of total perioperative costs. This is not to infer that the cost of anesthetic drugs should be ignored since they represent a small proportion of total perioperative costs; rather, it infers that we should prioritize our efforts for cost-savings interventions to be commensurate with the expected return on investment of effort. Specifically, since anesthetic drugs represent less than $6 \%$ of total perioperative costs, we should aim to find interventions that have a reasonable cost of implementation in order to achieve cost-savings that are worthy of the investment of our time and effort. The case of Tabing et al. ${ }^{2}$ is instructive since the intervention required a small investment of time and effort, given that it involved simply removing the costprohibitive drugs from easy access and limiting access by instituting a simple barrier. This simple intervention made an impact that might be considered modest yet perhaps worthy given the small up-front investment required. To sustain change management in perioperative practice, it will also be important to engage providers in shifting the culture of practice in the system.

\section{Value rather than cost}

The focus in healthcare should be the value rather than the price tag. If value is the goal, the most relevant question when choosing between anesthetic drugs is whether the most expensive agents provide a margin of benefit that is worthy of the additional cost. ${ }^{1}$ Meta-analyses found no difference in clinical or resource outcomes when comparing desflurane, dexmedetomidine, or remifentanil with active comparators. ${ }^{6-14}$ Many of the proposed statistically significant benefits of more costly anesthetic agents, such as faster emergence from anesthesia, do not translate into clinically important benefits in terms of real reductions in morbidity and resource utilization. 
This aligns with the findings of Tabing et al. ${ }^{2}$ wherein reducing access to the more costly agents had no measurable adverse effects on outcomes and allowed a reduction in the drug cost per case.

Most importantly, we should be strategic and proactive in identifying and implementing appropriate cost containment interventions rather than being primarily reactive or opportunistic, as in this study when desflurane was removed due to manufacturer recall or when critical drug shortages lead to reactive crisis management. Proactive and strategic approaches require that we systematically review the cumulating evidence and continuously estimate the relative cost effectiveness of our options while being open to disinvest from things that are no longer worthy of our limited resources. ${ }^{1}$

In this time of rapid growth in demand for healthcare, there is a pressing need to do all we can to justify the increasing costs and efforts expended to implement new drugs, technologies, and techniques into practice. ${ }^{19,20}$ Nevertheless, "we need to respect that we cannot (and should not) 'do it all', especially if the new techniques or technologies will achieve only marginal benefits at best and at greater risk and cost compared with the existing status quo." ${ }^{20}$ It is also important to remember that, given the relatively small proven differences between most drugs in head-to-head clinical trials, much of the time, what we give is not as important as how we give the anesthetic agents.

The importance of doing more within our set of available resources has taken central stage across the globe. This was highlighted by the Choosing Wisely international campaign on "how to do better with less" 21 and by the public drive by the Institute of Medicine (IOM) Roundtable on Value \& Science-Driven Health Care strategy to be fulfilled by 2020 . The goals of the study by Tabing et al. ${ }^{1}$ are also closely in line with the views of Sir Muir Gray, Chief Knowledge Officer, UK National Health Service, who recently ${ }^{\mathrm{A}}$ remarked: "Doing things right is only half the story-it is also essential to use our limited resources most effectively by identifying and discontinuing lower value activities".

\section{Le véritable coût des soins : viser l'optimisation de ressources avant de voir le coût}

Deux éléments sont probablement à l'origine de notre aversion naturelle à toute mesure de rationalisation des coûts : d'une part, nous avons peur que le coût supplante

\footnotetext{
A Gray JA. Better Value Health Care. Solutions: "Focus on Value'. Available from URL: http://www.bettervaluehealthcare.net (accessed July 2015).
}

toute considération d'efficacité; d'autre part, nous estimons souvent que le coût constitue un cadre de référence à la fois injuste, biaisé et déplaisant pour instruire des décisions cliniques où les enjeux sont - littéralement - des questions de vie (ou de membres). Ceci étant dit, on ne peut exclure toute considération financière de la prise de décision clinique si nous ne voulons pas aller droit au mur. En effet, une telle approche est fondée sur l'idée naïve que les ressources en santé sont illimitées. En réalité, si l'on ignore sciemment le coût des soins et interventions que nous prodiguons, nous perdrons davantage de vies et de membres qu'en nous fondant sur une approche raisonnée, où l'on tient compte de la valeur d'une intervention ou d'un médicament par dollar. Une approche de 'gratuité pour tous' dans la prise de décisions cliniques provoque une réduction de la valeur extraite de nos ressources.

Les ressources destinées aux soins de santé sont limitées; il faut donc faire des choix quant à ce qu'on utilise et ce à quoi on renonce. Même si l'on pouvait éliminer tout le gaspillage et nous limiter aux seules interventions qui 'fonctionnent' en soins de santé, la triste réalité demeure que les ressources à notre disposition ne suffisent pas pour répondre à tous les besoins - il faut donc, une fois de plus, faire des choix et renoncer à certaines choses au profit d'autres. Idéalement, ces décisions sont prises sur la base des meilleures données scientifiques possibles sur ce qui 'fonctionne' (soit la médecine factuelle), et sur quel éventail d'options offrira la plus grande valeur pour les patients tout en respectant les limites des ressources disponibles.

\section{Le rôle de l'analyse de l'économie de la santé}

Pour bien comprendre les initiatives de compression des coûts, il faut d'abord connaître les paramètres fondamentaux en économie de la santé : la minimisation des coûts, le rapport coût-efficacité, le rapport coût-utilité et le rapport coût-avantage. ${ }^{1}$ Plus spécifiquement, il faut comprendre que l'analyse des coûts en économie de la santé ne tient compte que d'un côté de l'équation. Lorsqu'il s'agit de soins de santé, l'analyse des coûts seulement ne suffit pas, car on est souvent d'accord pour payer toujours plus pour des résultats toujours meilleurs. C'est pourquoi on s'intéresse aux coûts $(\mathrm{C})$ et aux effets $(\mathrm{E})$, et surtout à savoir si l'augmentation des coûts est justifiée par les avantages supplémentaires ainsi procurés. L'analyse économique en santé exige qu'on calcule une équation de valeur afin d'estimer le coût-efficacité des autres options de soins. Cette équation peut être calculée par une analyse coût-efficacité (c.-à-d., 'quel coût supplémentaire est nécessaire pour obtenir un avantage supplémentaire pour le médicament A par rapport au médicament $\mathrm{B}$, exprimé en 
tant que $\Delta \mathrm{C} / \Delta \mathrm{E}$ ?') ou par une analyse coût-utilité (c.-à-d., 'quel coût supplémentaire est nécessaire pour obtenir une année de vie de qualité supplémentaire avec le médicament A par rapport au médicament $\mathrm{B}$, exprimé en tant que $\Delta \mathrm{C} /$ $\Delta$ QALY?'). ${ }^{1}$ L'analyse de minimisation des coûts, quant à elle, est une forme particulière d'analyse coût-efficacité dans laquelle les différentes options sont considérées comme étant équivalentes (et dans laquelle, par conséquent, $\Delta \mathrm{E}$ s'annule et devient zéro), et dans laquelle seule la différence en matière de coût demeure pertinente $(\Delta C)$.) Dans ce cas particulier d'équivalence, les coûts seuls sont utilisés pour prendre les décisions de coût-efficacité. Dans une analyse de minimisation des coûts, il est difficile de prouver l'équivalence, ou tout au moins de prouver qu'il n'existe pas de différence suffisamment grande pour être pertinente d'un point de vue clinique entre les médicaments.

En gardant ces principes à l'esprit, examinons l'étude de Tabing et coll. ${ }^{2}$ publiée dans ce numéro du Journal. Leur étude de cohorte décrit l'effet d'une mesure de restriction d'accès à trois médicaments peropératoires au coût relativement élevé (soit le rémifentanil, la dexmédétomidine et le desflurane) sur le coût des médicaments anesthésiques ainsi que sur certains résultats périopératoires choisis pour toutes les interventions chirurgicales pour lesquelles les données étaient disponibles. On compara rétrospectivement deux cohortes de patients : la première était composée des patients opérés avant la restriction d'accès au desflurane (une cohorte de commodité, définie par l'accès sans restriction à tous les agents en salle d'opération (SO) avant le rappel temporaire du fabricant des vaporisateurs de desflurane). La seconde cohorte était composée des patients pris en charge après la mise en place de la mesure de restriction d'accès aux médicaments anesthésiques coûteux (la restriction provenant de l'incommodité : une étape supplémentaire était requise de la pharmacie, qui devait noter le nom de l'anesthésiologiste supervisant le cas avant de lui procurer du rémifentanil, de la dexmédétomidine ou du desflurane). Le retrait par le fabricant du desflurane a servi d'élément déclencheur pour mettre en place une mesure destinée à réduire l'accès à cet anesthésique par inhalation plus dispendieux, ainsi qu'à deux autres agents intraveineux coûteux (le rémifentanil et la dexmédétomidine), pour lesquels il existe des alternatives moins chères. Comme on pouvait s'y attendre, en réduisant la facilité d'accès à ces agents plus dispendieux, leur utilisation a diminué et des économies ont ainsi été réalisées en matière de médicaments anesthésiques. De plus, aucun effet mesurable sur les résultats cliniques ou la durée d'hospitalisation n'a été observé.

Il ne fait aucun doute qu'en plaçant des obstacles à l'accès aux médicaments dispendieux, leur utilisation et les coûts des médicaments vont en général baisser. Toutefois, avant d'accepter aveuglément qu'une telle mesure soit appliquée dans d'autres contextes, il convient de réfléchir à plusieurs objections potentielles. Cette mesure de restriction est-elle appropriée et déontologique? En vaut-elle la peine? Comment pouvons-nous, de façon proactive, concentrer notre attention sur la 'valeur' plutôt que sur le 'coût' seul?

\section{Est-il acceptable de restreindre l'accès aux médicaments dispendieux?}

La restriction de l'accès suscite une aversion naturelle chez la plupart des cliniciens - après tout, comment de telles 'mesures administratives' peuvent-elles anticiper toutes les situations de patients auxquelles nous sommes confrontés en tant que cliniciens? Est-ce que certains patients, qui auraient profité des avantages du médicament restreint, n'y auront dorénavant plus accès? Et pourtant, une telle approche est fréquemment utilisée dans les formulaires de pharmacie (avec des effets prouvés sur les coûts des médicaments pour les hôpitaux et les programmes de médicaments financés par le gouvernement). ${ }^{3,4}$ De plus, la plupart des praticiens ne sont pas au fait des coûts, et encore moins du véritable coût-efficacité de la panoplie de médicaments parmi lesquels ils font leur choix. Dès lors, bien que notre préférence naturelle aille à un accès libre et sans restriction à tous les médicaments afin de nous permettre un choix sans restriction pour nos patients, un tel accès suppose que nous soyons capables de faire un choix éclairé parmi les différentes alternatives à notre disposition. En réalité cependant, nous choisissons sans avoir les informations nécessaires - autrement dit, sans connaître l'avantage supplémentaire prouvé et le coût supplémentaire des agents plus dispendieux par rapport aux alternatives disponibles.

Ce n'est pas parce qu'un médicament est disponible sur le marché qu'il représente nécessairement un progrès par rapport aux autres options, ni qu'il est rentable et devrait dès lors être systématiquement disponible en milieu hospitalier. L'existence d'un médicament sur le marché veut simplement dire qu'il a été jugé acceptable pour le marché - pas forcément qu'il est 'meilleur', ou qu'il 'vaut la peine' par rapport à ses alternatives. La restriction d'accès aux médicaments plus dispendieux peut être acceptable lorsque: (1) l'avantage supplémentaire du médicament plus dispendieux n'a pas été prouvé; ou (2) un léger avantage a été prouvé mais il n'est pas assez important pour justifier le coût supplémentaire; et (3) la restriction peut être levée lorsque cela est justifié (par ex. pendant une pénurie de médicaments, ou lorsqu'une situation clinique spécifique justifie véritablement la levée de cette restriction). La première condition semble facile à comprendre à prime abord, toutefois elle peut être 
difficile à remplir lorsqu'on sait que les études cliniques manquent souvent de puissance pour pouvoir prouver qu'il n'existe « aucune différence » et qu'elles ne peuvent que déclarer «qu'aucune différence n'a été observée ». Dans ce cas, «l'absence de données probantes n'est pas la preuve d'une absence ». ${ }^{5}$ Autrement dit, le manque d'études ayant suffisamment de puissance et une méthodologie suffisamment rigoureuse pour exclure toute différence pertinente d'un point de vue clinique entre des médicaments ne signifie pas forcément que les médicaments sont équivalents, seulement qu'on n'a pas pu prouver qu'ils étaient différents. On ne peut savoir si deux ou plusieurs médicaments donnés entraînent ou non des résultats différents, à moins d'avoir suffisamment d'études qui fournissent des intervalles de confiance suffisamment étroits pour exclure les différences suffisamment importantes pour qu'on en tienne compte.

Dans l'étude de Tabing et coll., ${ }^{2}$ il semble que la décision de restreindre l'accès à ces trois médicaments était fondée d'une part sur une considération de coûts, et d'autre part sur le fait qu'aucun avantage n'ait été prouvé par rapport aux alternatives moins dispendieuses à la disposition des cliniciens (ou que les preuves d'avantages suffisamment importants manquaient pour justifier le coût significativement plus élevé). En règle générale, les études randomisées et les méta-analyses concernant l'utilisation du rémifentanil, de la dexmédétomidine et du desflurane en SO n'ont pu en prouver la supériorité par rapport à leurs alternatives moins dispendieuses. ${ }^{6-14}$ Qui plus est, l'obstacle érigé pour restreindre l'accès aux trois médicaments était minime (garder les médicaments hors de portée immédiate et demander que l'anesthésiologiste s'identifie avant de faire sortir le médicament de la pharmacie de SO). Il est toutefois impossible de déterminer si ce modeste obstacle suffirait à maintenir, à long terme, une réduction de l'utilisation inappropriée de ces agents.

\section{La restriction d'accès est-elle déontologique?}

Les objections déontologiques à la restriction de l'accès à certains médicaments sont difficiles à faire valoir lorsque les données probantes convaincantes concernant leur supériorité font défaut. Ces objections peuvent tout de même exister lorsqu'il s'agit de cas véritablement 'exceptionnels', dans lesquels le manque de données probantes est dû à la rareté de l'application ou de la condition; une utilisation du médicament unique et spécifique au patient pourrait alors être justifiée si les alternatives raisonnables ne fonctionnent pas ou sont contre-indiquées.

Certaines objections déontologiques pourraient également être soulevées par les institutions d'enseignement. En effet, est-il justifié de limiter l'accès aux médicaments plus récents si notre mandat est d'enseigner? Dans un centre d'enseignement, le maintien de l'accès à un large éventail de médicaments anesthésiques est important, que ce soit à des fins d'enseignement ou de recherche. Ces éléments ne sont, en règle générale, pas pris en compte dans les analyses de coût-efficacité; par conséquent, il serait utile de trouver des manières d'intégrer ces 'mesures intangibles' de façon plus explicite dans notre évaluation des impacts liés aux restrictions d'accès. En réfléchissant à ces questions, il peut également être intéressant d'envisager la restriction d'accès comme une bonne occasion d'enseigner à nos étudiants à s'impliquer dans la gestion intelligente des ressources et à être capable de se débrouiller dans un système où seuls certains médicaments sont disponibles. De plus, le nombre toujours croissant de médicaments, d'appareils et de technologies, a créé une situation dans laquelle il est impossible d'être au fait de tous les médicaments et technologies pertinents, aujourd'hui et demain. Plutôt que d'essayer tant bien que mal d'exposer nos étudiants à tous les agents coûteux, peut-être devrions-nous envisager un nouveau paradigme : enseigner aux étudiants et aux cliniciens comment maintenir leurs connaissances et compétences à jour dans un environnement en évolution constante, et leur offrir des occasions de se familiariser avec les agents inaccessibles par des formations vidéo proactives sur demande, ainsi que par des simulations innovantes. En fait, avec l'expansion constante du marché, le problème ne va qu'empirer au fil du temps.

Le fait de réduire les médicaments anesthésiques à leur plus faible dénominateur de coût global dans les soins chirurgicaux périopératoires pourrait avoir des conséquences inattendues, comme par exemple de contribuer aux pénuries de médicaments. ${ }^{15}$ Par exemple, les médicaments plus anciens sont souvent les plus rentables à utiliser, mais du point de vue du fabricant, ces 'vieux' médicaments sont aussi les moins profitables. De plus, dans une société de libre entreprise, les gouvernements ne peuvent promulguer des lois qui obligent les compagnies à fabriquer certains produits. Les médicaments et anesthésiques génériques sont donc vulnérables aux interruptions d'approvisionnement et aux pénuries, en raison de la concentration du marché, comme ce fut récemment le cas avec Sandoz et Hospira, qui fournissent $80 \%$ des médicaments injectables génériques utilisés en milieu hospitalier. ${ }^{16}$ Un nouveau médicament peut prendre dix à quinze ans à mettre au point, avec un coût moyen (y compris les échecs) de plus de 2,6 milliards $\$$ pour l'industrie pharmaceutique. ${ }^{17}$ Les agents anesthésiques sont souvent au bas de la liste des priorités de l'industrie pharmaceutique, car le retour-sur-investissement est bien plus élevé dans d'autres secteurs. Tout bien considéré, nous devons également tenir compte du fait qu'un élément clé pour motiver et soutenir l'innovation est de payer le bon prix plutôt que d'essayer à tout prix de le réduire au minimum. 


\section{La restriction d'accès 'vaut-elle vraiment la peine'?}

Pour être considérées comme 'valables', les économies ainsi faites doivent avoir plus de valeur que ce à quoi on renonce, y compris la liberté de choix réduite, et tous les coûts et efforts supplémentaires nécessaires à mettre en œuvre et maintenir la mesure de réduction des coûts. La chirurgie est indubitablement une entreprise dispendieuse lorsqu'on considère ses coûts directs en proportion des dépenses hospitalières totales, mais il n'existe que peu d'analyses formelles concernant le coût de la SO. Une étude examinant les coûts liés à la chirurgie et réalisée dans un contexte de soins de santé privés aux États-Unis a rapporté que les SO étaient à l'origine de $40 \%$ des dépenses hospitalières totales, mais qu'elles généraient 70 $\%$ des revenus. ${ }^{18}$ Le coût des médicaments (anesthésiques et non anesthésiques) constituait $6 \%$ du coût hospitalier total; les médicaments anesthésiques, eux, représentaient $22 \%$ du coût total des médicaments hospitaliers. ${ }^{18}$ Cependant, en calculant le coût total de la SO, les médicaments anesthésiques peropératoires n'ont constitué que $5,6 \%$ des coûts périopératoires totaux. Cela ne veut pas dire qu'il faut ignorer le coût des médicaments anesthésiques parce qu'ils ne représentent qu'une petite partie des coûts périopératoires totaux; plutôt, nous devrions prioriser nos efforts en matière de mesures de compression budgétaire, afin que les résultats soient proportionnels aux efforts investis. De plus, étant donné que les médicaments anesthésiques représentent moins de 6 \% des coûts périopératoires, il faut que le coût de mise en œuvre de nos interventions demeure raisonnable, afin que les économies réalisées soient dignes de nos investissements en temps et en efforts. L'étude de Tabing et coll. ${ }^{2}$ est utile; en effet, la mesure n'a nécessité qu'un faible investissement en matière de temps et d'effort : il a simplement fallu rendre un peu plus difficile l'accès aux médicaments dispendieux en érigeant un simple obstacle. Cette mesure simple a eu un impact, qui pourrait certes être perçu comme modeste, mais peut-être valable, étant donné le petit investissement nécessaire à la base. Si l'on souhaite gérer à long terme les changements de la pratique périopératoire, il est important d'impliquer les intervenants, car ce sont eux qui, en fin de compte, feront évoluer la culture de la pratique.

\section{La valeur plutôt que le coût}

En soins de santé, l'emphase devrait être mise sur la valeur plutôt que sur l'étiquette de prix. Si l'objectif est la valeur, la question la plus pertinente lorsqu'on choisit un médicament anesthésique est de savoir si l'agent plus dispendieux offre un avantage supplémentaire qui justifie la différence de prix. ${ }^{1}$ Les méta-analyses publiées à ce jour n'ont pas trouvé de différence en matière de résultats cliniques ou de ressources lors de comparaisons entre le desflurane, la dexmédétomidine ou le rémifentanil et des agents comparables. ${ }^{6-14}$ Plusieurs avantages significatifs d'un point de vue statistique des agents anesthésiques plus dispendieux, tels qu'un réveil plus rapide après l'anesthésie, ne se traduisent pas en avantages importants d'un point de vue clinique en termes de réductions réelles de la morbidité ou de l'utilisation des ressources. Ces résultats vont dans le même sens que ceux de Tabing et coll., ${ }^{2}$ où la réduction de l'accès aux agents plus dispendieux n'a pas eu d'effet indésirable mesurable sur les résultats, et a permis de réduire le coût des médicaments par cas.

Il serait plus important de faire preuve de stratégie et d'être proactifs en identifiant et en mettant en œuvre des mesures adaptées de maîtrise des coûts, plutôt que d'être réactifs et opportunistes, comme ce fut le cas dans cette étude, où le desflurane a été retiré en raison d'un rappel du fabricant, ou lorsque des pénuries de médicaments graves entraînent une gestion de crise en réaction. $\mathrm{Si}$ nous souhaitons être proactifs et stratégiques, nous devons systématiquement passer en revue les données qui s'accumulent et constamment estimer le rapport coût-efficacité relatif de nos options, tout en étant ouverts à l'abandon de certains éléments qui n'ont plus leur place dans l'allocation de nos ressources limitées. ${ }^{1}$

En cette époque de croissance rapide de la demande en soins de santé, il est urgent de faire tout notre possible pour justifier les coûts et les efforts croissants pour mettre en œuvre de nouveaux médicaments, de nouvelles technologies et techniques dans la pratique. ${ }^{19,20}$ Toutefois, «il nous faut respecter que nous ne pouvons pas (et ne devrions pas) tout faire, particulièrement si les nouvelles techniques et technologies n'apportent que des avantages marginaux au mieux, et engendreront des risques et des coûts plus élevés que le statu quo existant. ${ }^{20}$ Il faut également garder à l'esprit que, étant donné les différences relativement petites entre la plupart des médicaments dans les études cliniques de comparaison directe, ce ne sont pas les agents que nous administrons qui sont si importants - mais plutôt la façon dont nous les administrons.

L'importance de faire plus avec les ressources à notre disposition est de plus en plus prépondérante dans le monde, comme le souligne la campagne Choosing Wisely International dont l'objectif est de montrer «comment faire mieux avec moins $» .^{21}$ Le but ultime de cette campagne est la motivation du public à atteindre, d'ici 2020, une utilisation de plus en plus responsable des ressources en santé, à l'aide d'une Table ronde de l'Institut de médecine sur la valeur des soins de santé fondés sur la 
science («Institute of Medicine Roundtable on Value and Science-Driven Healthcare Strategy »). Les objectifs de l'étude de Tabing et coll. ${ }^{1}$ s'alignent également avec ceux de Sir Muir Gray, responsable des connaissances des Services de santé nationaux britanniques (NHS), qui remarquait récemment: ${ }^{\mathrm{A}}$ "Bien faire les choses n'est que la moitié de l'histoire - il est également essentiel d'utiliser nos ressources limitées de la façon la plus efficace qui soit en identifiant et en cessant les activités de moindre valeur».

Conflicts of interest None declared.

\section{Conflit d'intérêt Aucun.}

Open Access This article is distributed under the terms of the Creative Commons Attribution-NonCommercial 4.0 International License (http://creativecommons.org/licenses/by-nc/4.0/), which permits any noncommercial use, distribution, and reproduction in any medium, provided you give appropriate credit to the original author(s) and the source, provide a link to the Creative Commons license, and indicate if changes were made.

\section{References}

1. Martin J, Cheng D. Role of anesthesiologists in the wider governance of health care and health economics. Can J Anesth 2013; 60: 918-28.

2. Tabing AK, Ehrenfeld JM, Wanderer JP. Limiting the accessibility of cost-prohibitive drugs reduces overall anesthetic drug costs: a retrospective before and after analysis. Can J Anesth 2015; 62: this issue. DOI:10.1007/s12630-015-0442-8.

3. Sullivan SD, Yeung K, Vogeler C, et al. Design, implementation, and first-year outcomes of a value-based drug formulary. J Manag Care Spec Pharm 2015; 21: 269-75.

4. Persson EL, Miller KS, Nieman JA, Sgourakis AP, Akkerman SR. Formulary evaluation using a class review approach: experience and results from an academic medical center. P T 2013; 38: 213-6.

5. Sagan $C$. The Demon-Haunted World: Science as a Candle in the Dark. 1st ed. NY: Ballantine; 1997. p. 213.

6. Gupta A, Stierer T, Zuckerman R, Sakima N, Parker SD, Fleisher $L A$. Comparison of recovery profile after ambulatory anesthesia with propofol, isoflurane, sevoflurane and desflurane: a systematic review. Anesth Analg 2004; 98: 632-41.

7. White PF, Tang J, Wender RH, et al. Desflurane versus sevoflurane for maintenance of outpatient anesthesia: the effect on early versus late recovery and perioperative coughing. Anesth Analg 2009; 109: 387-93.

8. Elliott RA, Payne K, Moore JK, et al. Which anaesthetic agents are cost-effective in day surgery? Literature review, national survey of practice and randomised controlled trials. Health Technol Assess 2002; 6: 1-264.

9. Greco M, Landoni G, Biondi-Zoccai $G$, et al. Remifentanil in cardiac surgery: a meta-analysis of randomized controlled trials. J Cardiothorac Vasc Anesth 2012; 26: 110-6.

10. Komatsu R, Turan AM, Orhan-Sungur M, McGuire J, Radke OC, Apfel CC. Remifentanil for general anaesthesia: a systematic review. Anaesthesia 2007; 62: 1266-80.

11. Howie MB, Cheng DC, Newman MF, et al. A randomized doubleblinded multicenter comparison of remifentanil versus fentanyl when combined with isoflurane/propofol for early extubation in coronary artery bypass graft surgery. Anesth Analg 2001; 92: 1084-93.

12. Cheng $D C$. Fast track cardiac surgery pathways; early extubation, process of care, and cost containment. Anesthesiology 1998; 88: 1429-33.

13. Le Bot A, Michelet D, Hilly J, et al. Efficacy of intraoperative dexmedetomidine compared with placebo for adult surgery: a meta analysis of published studies. Minerva Anestesiol 2015 (in press).

14. Wijeysundera DN, Bender JS, Beattie WS. Alpha-2 adrenergic agonists for the prevention of cardiac complications among patients undergoing surgery. Cochrane Database Syst Rev 2009; 4: CD004126.

15. Hall $R$, Chisholm $R$, Cheng D, Murphy M, Campbell D. Drug shortages in anesthesia and perioperative medicine: Canada needs a better supply system. Can J Anesth 2012; 59: 629-35.

16. ASHP. Drug Shortages. Current Drugs. Available from URL: http://www.ashp.org/DrugShortages/ (accessed July 2015).

17. Mullard A. New drugs cost US $\$ 2.6$ billion to develop. Nature Reviews Drug Discovery 2014; 13: 877.

18. Macario A, Vitez TS, Dunn B, McDonald T. Where are the costs in perioperative care? Analysis of hospital costs and charges for inpatient surgical care. Anesthesiology 1995; 83: 1138-44.

19. Bainbridge D, Martin J, Arango M. Cheng D; Evidence-Based Peri-operative Clinical Outcomes Research (EPICOR) Group. Perioperative and anaesthetic-related mortality in developed and developing countries: a systematic review and meta-analysis. Lancet 2012; 380: 1075-81.

20. Cheng D, Martin J. Evidence-based practice and health technology assessment: a call for anesthesiologists to engage in knowledge translation. Can J Anesth 2011; 58: 354-63.

21. Levinson W, Kallewaard M, Bhatia S, Wolfson D, Shortt S, Kerr E; Choosing Wisely International Working Group. 'Choosing Wisely': a growing international campaign. BMJ Qual Saf 2015; 24: $167-74$. 